\title{
Origen de los problemas administrativos estructurales empresariales con enfoque sistémico
}

\section{Origin of business structurals administratives problems with a systemic approach}

Iván Vaca Aguirre* ivacaa@ups.edu.ec

\begin{abstract}
Resumen
Las ideas sobre sistemas abiertos del biólogo y filósofo austríaco Ludwig Von Bertalanffy, presentadas en 1950 en su Teoría General de Sistemas han sido aplicadas a la teoría organizacional, en base a la concepción de que las organizaciones constituyen sistemas abiertos. Bajo esta concepción sistémica, se entiende que la empresa es un sistema abierto que interactúa con su entorno.

En este contexto, la finalidad de este artículo es dar a comprender que la empresa es un sistema abierto que interactúa con su entorno, hacer conocer cuáles son los elementos constituyentes del sistema empresa y dar a entender que, en su condición sistémica, la inadecuada interacción de sus elementos ocasionaría problemas en la estructura del sistema y no de disfunciones de las partes consideradas aisladamente.
\end{abstract}

\section{Palabras clave}

Sistemas, empresa, estructura administrativa, organización y gestión. 


\begin{abstract}
Ideas about open systems biologist and Austrian philosopher Ludwig Von Bertalanffy, presented in 1950 in his General Theory of Systems have been applied to organizational theory, based on the notion that organizations are open systems. Under the systemic approach, it is understood that the company is an open system that interacts with its environment.

In this context, the purpose of this paper is to understand that the company is an open system that interacts with its environment, to know what the constituent elements of the business system and to suggest that, in its systemic condition, inadequate interaction of its elements would cause problems in the structure of the system and not of dysfunctions of the parties considered in isolation.
\end{abstract}

\title{
Keywords
}

Systems, company, management structure, organization and management.

Forma sugerida de citar: Vaca Aguirre, Iván Patricio (2015). Origen de los problemas administrativos estructurales empresariales, con enfoque sistémico. Retos, 10(1), pp.193-204.

\section{Introducción}

Para Peter Senge: Un sistema es una totalidad percibida cuyos elementos se "aglomeran" porque se afectan recíprocamente a lo largo del tiempo y operan con un propósito común (Senge, 2006).

Por su parte, los autores (Hernández \& Pulido, 2011: 24), definen a los sistemas como "Un conjunto de elementos íntimamente relacionados para un fin, estructurados por subsistemas y delimitados por el entorno o macrosistema en que actúan".

El autor Rodríguez, refiriéndose a los sistemas abiertos manifiesta:

El sistema abierto está en interacción continua con su medio y logra un estado estable o de equilibrio dinámico, mientras conserva su capacidad de trabajo o de transformación de energía. En efecto, la supervivencia del sistema no sería posible sin un flujo continuo de transformación y producción.

Por ejemplo, un organismo social recibe insumos de la sociedad en forma de personal, material, dinero, información; transforma esos insumos en productos y servicios, y a su personal para mantener su participación. Para el organismo, el dinero y el mercado proveen el mecanismo para reiniciar el ciclo de recursos entre ella y su ambiente (Rodríguez Valencia, 2002: 7).

En este contexto, los autores (Robbins \& Coulter, 2010: 35), refieren: “... los sistemas abiertos se ven influenciados por su entorno e interactúan con él. Hoy en día, cuando describimos a las organizaciones como sistemas, nos referimos a sistemas abiertos". 
Con relación a la empresa como sistema, Laborda \& De Zuani (2005), afirman:

La interacción de la empresa con el ambiente permite destacar una de las perspectivas más importantes bajo las que se ha estudiado la empresa como organización, y que es conocida como el enfoque de sistemas, a partir del cual la empresa es considerada como un sistema abierto, regido por principios, que presenta unas características que lo configuran como un sistema complejo (Laborda Castillo \& De Zuani, 2005: 35).

\section{En el campo empresarial:}

Este enfoque sistémico es la quinta disciplina de las cinco propuestas por el profesor Peter Senge, en su libro La quinta disciplina, siendo la más importante que deben seguir las organizaciones para tener éxito en una época de nuevos y constantes retos (El Norte, 1997).

De lo anterior, se evidencia que: los sistemas se caracterizan por el conjunto de partes y componentes -elementos- que se encuentran organizados y relacionados, con el propósito de lograr un objetivo común. Los sistemas, poseen organización interna y estructura que integra y mantiene unida las partes dando lugar a la propiedad holística del sistema. Concebido un sistema en el cual los elementos que lo constitu- yen están relacionados entre sí (son interdependientes), tienen un propósito común y su interacción funciona de tal manera que, cualquier cambio, en cualquiera de ellos, afecta al conjunto.

Ante la posible falta de relación de los elementos del sistema empresa, surge la pregunta de investigación: ¿De qué manera afecta a la empresa la falta de interrelación de los elementos que la componen?

\section{Hipótesis}

La falta de relación entre los elementos del sistema empresa afecta a su organización, el sistema falla y no se cumplen con los objetivos empresariales.

\section{Objetivo General}

Determinar en dónde se originan los problemas administrativos estructurales empresariales basado en un enfoque sistémico, mediante un estudio de fuentes bibliográficas.

\section{Materiales y métodos}

$\mathrm{Al}$ proponer este trabajo cuyo propósito principal es comunicar resultados empíricos, se realiza un estudio de fuentes bibliográficas considerando libros y publicaciones académicas, relacionados con el concepto de sistemas y el enfoque sistémico de la empresa.

La selectividad de las fuentes de consulta se realiza de aquellas que 
describen al sistema empresa con sus características, elementos y estructura y de las que se enfocan en los problemas estructurales de la empresa.

La metodología considera además la utilización y revisión de fuentes secundarias de información académica con la finalidad de sustentar el concepto sistémico de la empresa, la descripción de sus elementos y la importancia de su relación.

Como parte de la metodología se da respuesta a la pregunta ¿De qué manera afecta a la empresa la falta de interrelación de los elementos que la componen?, como propósito de analizar la importancia de mantener la calidad de las relaciones de los elementos del sistema empresa y así lograr los objetivos empresariales.

\section{Resultados}

\section{Sistemas, propiedades y relaciones}

En referencia a los sistemas, para los autores Arias \& Heredia (2012):

Un sistema puede definirse como un conjunto de diversos elementos, mismos que se encuentran interrelacionados. Nótese que el punto clave de un sistema está constituido por las relaciones entre los diversos elementos del mismo. Puede existir un conjunto de objetos; pero si éstos no están interconectados de alguna manera, entonces no constituyen un sistema" (Arias Galicia \& Heredia Espinosa, 2012: 107-108).
Cada sistema se encuentra en un medio circundante (suprasistema). De la misma manera que existen relaciones entre los diversos elementos del sistema, puede existir tales nexos entre el sistema y el suprasistema; se dice, entonces, que el sistema es abierto (p. 110).

Además, los sistemas tienen propiedades comunes que ayudan a entender la realidad en términos sistémicos, destacando que los sistemas:

- Cumplen con una misión o razón de ser;

- Tienen una tendencia natural a la desorganización o entropía;

- Cuentan con mecanismos de retroalimentación para contrarrestar la entropía;

- Mediante la retroalimentación, los sistemas disminuyen la tendencia a la entropía, generando una tendencia al equilibrio dinámico u homeostasis;

- Consumen alguna forma de energía;

- Se ubican en un entorno o ambiente que debe proporcionarle los insumos necesarios;

- Evolucionan e integran mayor complejidad. Si no evolucionan desaparecen o caen en la entropía;

- Están formados por elementos que a su vez constituyen sistemas. Al respecto, Mario Ernesto Martínez Avella (2005) en su obra Ideas para el cambio y el aprendizaje en la organización, menciona: 
El paradigma de sistemas constituye una de las formas de pensamiento más avanzadas en la comprensión de la dinámica de las organizaciones y el diseño de modelos de gestión para las empresas modernas. Sus ideas, desarrolladas durante más de cincuenta años, han regresado con fuerza a las teorías administrativas, tanto por su relación con el complejo mundo de la tecnología y la información, como por su utilización en las modas que nos dejaron las décadas pasadas y que hoy son objeto de estudio fundamental en las escuelas de negocios (Martínez, 2005).

Este autor, en la obra citada, refiriéndose al nuevo concepto de sistema, dice:

Los sistemas serían distinciones que hace un observador al atribuir significado a una serie de elementos organizados que se entrelazan en una red de relaciones efectivas para cumplir con un propósito, y en aquello que el observador sistémico distingue, resultan entonces prioritarios el contexto y las relaciones, más que los objetos en sí mismos (2005: 12).

De lo mencionado por los autores Arias Galicia Fernando y Mario Ernesto Martínez, se destaca la importancia que asignan a las relaciones entre los elementos organizados del sistema que, según Mario Martínez, se entrelazan en una red de relaciones efectivas para cumplir con un propósito. En el caso del sistema empresa, el propósito bien podría ser el cumplimiento de los objetivos estratégicos.

Bertalanffy (2007), en la introducción de su obra Teoría General de los Sistemas, publicado en 1968, en su obra original General System Theory; Foundation Development, Applications, con base en la teoría de las organizaciones formales que considera a las empresas de negocios como tal, refiere la premisa de que el único modo significativo de estudiar la organización es estudiarla como sistema. Entendiendo al sistema, según este autor, como un complejo de elementos interactuantes (Bertalanffy, 2007: 7-56).

El concepto sistémico implica que se debe enfrentar situaciones dinámicas inciertas y con frecuencia ambiguas que afectan al sistema. En este contexto, cuando se afecta a la calidad de las relaciones de los elementos y componentes del sistema empresa, éstos no están relacionados, no constituyen un sistema, el sistema falla, y no se cumplen con los objetivos empresariales. Con este enfoque, dada la condición sistémica de la empresa la inadecuada interacción de sus elementos ocasionaría problemas de estructura en la empresa.

Respecto a las interacciones, según Arévalo:

Las organizaciones empresariales son sistemas complejos, dado que su comportamiento se explica más en función de las interacciones que de las acciones de los agentes. Las interacciones facilitan el surgi- 
miento de condiciones novedosas que son absorbidas por el sistema promoviendo su evolución; es decir, el sistema no solo se acomoda a las condiciones cambiantes, sino que se transforma y modifica el entorno. Dado lo anterior, en el contexto de la administración se sugiere el uso de la expresión sistema complejo más que sistema adaptativo complejo (Arévalo Bohórquez, 2013).

\section{Discusión de resultados}

\section{Elementos e interacciones del sistema empresa}

(Luhmann, 1997), en su obra Sociedad y sistema: la ambición de la teoría, publicado en su obra original System and Funktion, propone el concepto de sistema autorreferente ${ }^{1} \mathrm{y}$ autopoiético. En la teoría de los sistemas autorreferentes, el sistema se define, por su diferencia respecto a su entorno; una diferencia que se incluye en el mismo concepto de sistema. El sistema, que contiene en sí mismo la diferencia con su entorno, es un sistema autorreferente y autopoiético. En lo autopoiético o autocreación, introduce las aportaciones de la denominada teoría de la autopoiesis, elaborada por los biólogos chilenos

1 En referencia al sistema, cuando él mismo constituye los elementos que le dan forma como unidades de función, y cuando todas las relaciones entre estos elementos van acompañadas de una indicación hacia esta autoconstitución, reproduciéndose de esta manera la autoconstitución permanentemente (Luhmann, 1997, 91).

Retos V (I0): 2015

(C) 2015, Universidad Politécnica Salesiana del Ecuador
H. Maturana y F. Varela. Según esta teoría, un sistema es autopoiético en tanto es un sistema que puede crear su propia estructura y los elementos de que se compone (p.18-19).

En el contexto de la autopoiesis, según (Maturana \& Varela, 1996: 36), los seres vivos se caracterizan porque se producen a sí mismos continuamente con una organización que los define organización autopoiética explicados en términos de relaciones. En este sentido, (Limone \& Cademártori, 1998, 23), en su libro La empresa una red de transformaciones señalan que: "la empresa puede ser considerada un sistema autopoiético de actos en el dominio de interacciones humanas. El dominio de existencia de la empresa es una intersección del dominio de relaciones laborales, tecnológicas y económicas que surgen en el espacio de las relaciones humanas...".

Retomando a Luhmann, desde el punto de vista de la investigación sociológica, en el contexto de la teoría general de sistemas, las relaciones entre elementos pueden condicionarse mutuamente. Existe una cuando otra también existe (1997: p. 66).

Por su parte (Mayntz, 1972: 52 53), en reimpresión de su obra original Sociologie der Organisation (1963), partiendo del supuesto de que "todas las formaciones sociales que poseen las características de la definición de organización tienen determinados problemas comunes y por ello pueden ser también acometidos con los 
mismos planteamientos", afirma: "En el análisis de la organización todas las organizaciones son consideradas como sistemas sociales encaminados a ciertos objetivos".

\section{Para este autor:}

Un sistema es un todo integrado por elementos que guardan entre sí una relación recíproca. La naturaleza especial de sus respectivos elementos, su modelo de ordenación y las relaciones entre ellos condicionan la peculiaridad concreta de un sistema. Dentro de un sistema, la variación de un elemento repercute sobre los demás elementos. Un sistema posee una cierta medida de integración y de hermeticidad (Mayntz, 1972).

En este sentido, se podría afirmar que el cumplimiento de los objetivos estratégicos empresariales dependerá de la calidad de las relaciones de los diferentes elementos y componentes del sistema organizacional denominado empresa. Cuando se afecta a la calidad de las relaciones de los elementos y componentes, a decir del autor Arias Fernando, “... pero si éstos no están interconectados de alguna manera, entonces no constituyen un sistema”.

Los profesores Limone y Cademártori (1998) señalan:

La empresa ha sido conceptualizada como una red de procesos de producción de componentes laborales, tecnológicos y económicos, los que en sus interacciones recurrentes vuelven a generar continua y recur- sivamente esa misma red de procesos que los produjo a ellos (p. 23).

\section{Continúan:}

Como unidad compuesta, la empresa tiene organización y estructura. Su organización autopoiética $^{2}$ queda definida por un conjunto de relaciones de especificidad, constitutivas y de orden (Maturana y Varela, 1972) que producen, relacionan y concatenan actos laborales, tecnológicos y económicos, generando una trama que puede materializarse como unidad topológica en el dominio de existencia de esos componentes laborales, tecnológicos y económicos (espacio de relaciones humanas).

$\mathrm{Su}$ estructura se constituye en las interacciones de elementos humanos, materiales, simbólicos y energéticos, las que producen actos laborales, tecnológicos y económicos concatenados de tal manera que materializan instante a instante la organización empresa en el dominio de interacciones de interacciones humanas en el que tales actos surgen y contribuyen a constituir (espacio de relaciones humanas) (Limone y Cademártori, 1998: 24).

2 Autopoiésis, del griego auto = sí mismo, y poios $=$ crear o producir.

Aplicado a la empresa, concepción de la empresa como un ente similar a un ser vivo y capaz de adquirir conocimiento. Según biólogos chilenos Maturana y Varela, un ser vivo es una máquina que se fabrica a sí misma permanentemente o una máquina cuyo funcionamiento permite la mantención de su propia organización en el tiempo (Maturana, H.; Varela, F., 1996). 
De lo mencionado por estos profesores, se aprecia que la empresa tiene organización, estructura y un conjunto de elementos de tipo humano, materiales, simbólicos (información) y energético (recursos financieros) y se infiere que los problemas de los sistemas resultan de las interacciones que se producen en un sistema, y no de disfunciones de las partes consideradas aisladamente, convirtiéndose en problemas estructurales sistémicos.

Según Limone y Cademártori: La organización se entenderá el conjunto de relaciones que define y determina las condiciones de constitución y funcionamiento de un sistema como unidad, esto es, su identidad de clase y su dinámica invariante. La estructura, es la forma como la organización se realiza efectivamente en una unidad concreta (p. 26).

El conjunto de elementos constituyentes está integrado por los recursos humanos, recursos materiales, la información y los recursos financieros.

A través de las interacciones recurrentes y recursivas de los elementos humanos, materiales, simbólicos y energéticos que integran el sistema, genera la trama de relaciones laborales, tecnológicas y económicas concatenadas que materializa la organización empresa, en el dominio de relaciones humanas, mediante la producción continua e interrumpida, en esos procesos, de las mismas relaciones (Limone y Cademártori, 1998: 33).

Las relaciones laborales (o de trabajo) (R.L.) son un tipo de interacciones humanas que se constituyen en un acuerdo para la realización de una tarea o grupo de tareas, y como tal, esas interacciones se dan en un dominio de acciones especificado por la emoción básica del compromiso (p. 39).

Las relaciones tecnológicas (R.T.) son interacciones que tienen que ver con el hacer y la modificación del hacer. Como tales, se dan en la interacción de los seres humanos con otros seres humanos y preponderantemente con los objetos (p. 39).

Las relaciones económicas (R.E.) son un tipo de interacciones que se dan en el espacio de las relaciones humanas y que tienen directa implicancia en la constitución y realización de la empresa (p. 40). 
Figura 1. Elementos, Interacciones, Relaciones

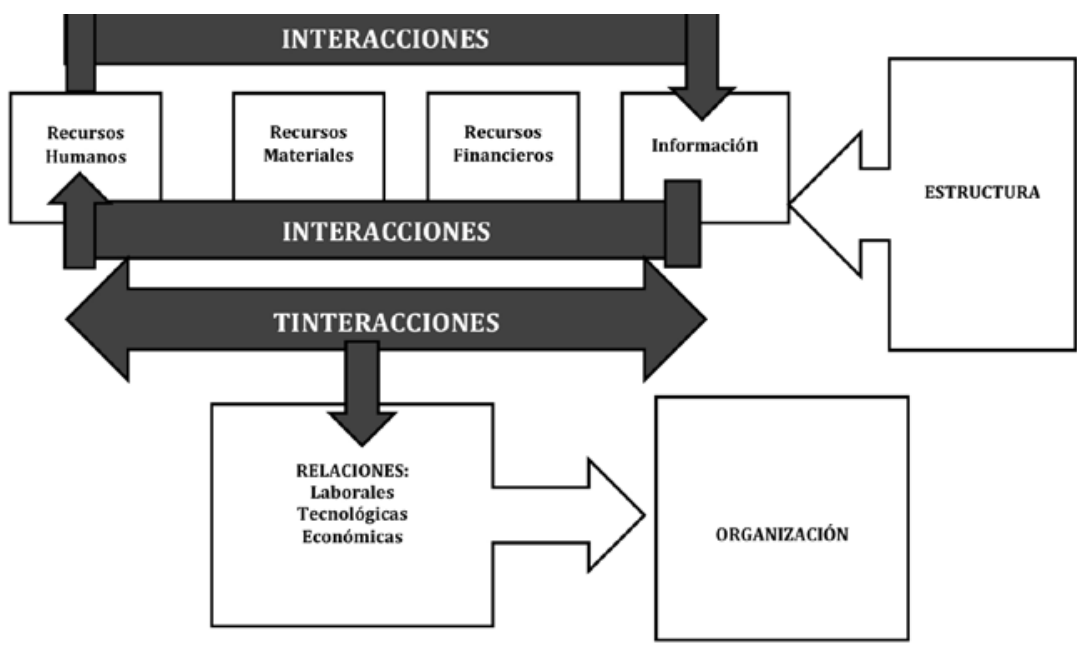

Fuente: Elaboración propia

En tal virtud, tomando de referencia lo expuesto por los profesores Limone y Cademártori, y en relación a la pregunta planteada en la Introducción de este artículo, ¿De qué manera afecta a la empresa la falta de interrelación de los elementos y componentes del sistema empresa?, se deduce que las causas de los problemas estructurales sistémicos en la empresa que afectan a la organización y estructura empresarial y no permiten el cumplimiento de sus objetivos estratégicos, desde el punto de vista sistémico, se originan en la calidad de las relaciones laborales, relaciones tecnológicas y relaciones económicas que materializan la organización empresa a través de las interacciones recurrentes y recursivas de sus elementos constituyentes de tipo humano, material, información y recursos financieros que son parte constitutiva de la estructura de la empresa.

Lo mencionado, encuentra sustento en lo referido por los autores Arias Galicia y Heredia Espinosa (2012) quienes afirman, "el punto clave de un sistema está constituido por las relaciones entre los elementos. Puede existir un conjunto de objetos, pero si éstos no están relacionados de alguna manera, entonces no constituyen un sistema”.

En referencia a los problemas de los sistemas sociales dirigidos a un objetivo, Maintz (1972), asegura: "El primer problema fundamental se 
refiere a las dependencias y acciones recíprocas entre los elementos, especialmente entre los factores analíticos del sistema social de la organización” (Mayntz, 1972: 63).
De allí que, para solucionar problemas sistémicos, en la mayoría de las ocasiones, es más eficaz (y más fácil) actuar sobre las relaciones que sobre los elementos de un sistema.

Figura 2. Efecto de la calidad de las Relaciones laborales, tecnológicas y económicas

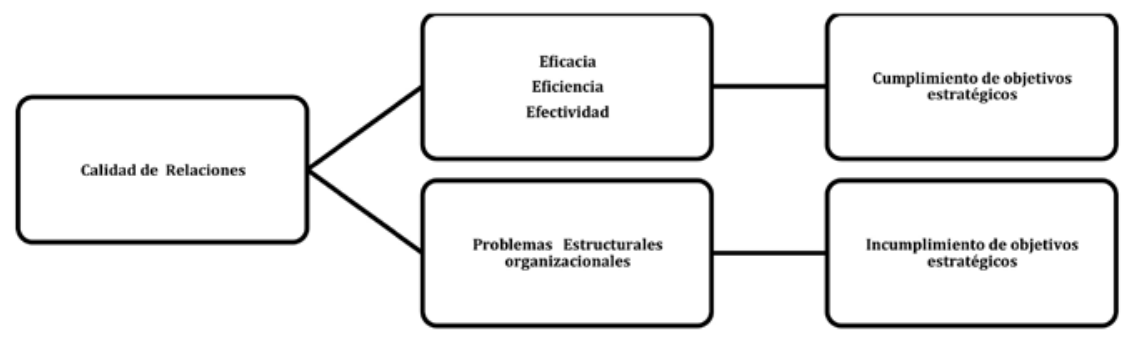

No obstante, para disminuir los problemas estructurales organizacionales ¿Cómo se podría mantener la calidad de las relaciones?; y, ¿Cuáles serían los principales aspectos a controlar de las relaciones?, constituyen interrogantes adicionales que también podrían ser analizadas con enfoque sistémico.

En este contexto, refiriéndose a los estudios organizacionales (EO), Gonzáles-Miranda (2014), en su documento introductorio "Estudios Organizacionales. Un campo de conocimiento comprensivo para el estudio de las organizaciones", entre las conclusiones afirma:

Si bien los EO están todavía en construcción y muchos investigadores se acercan a él con definiciones aún inacabadas, es posible afirmar que se trata de una nueva perspectiva para la comprensión de los fenómenos organizacionales, cuya riqueza se encuentra en esa pluridisciplinariedad y en su perspectiva crítica, aspectos relevantes tanto para la investigación como para la intervención organizacional (González-Miranda, 2014).

\section{Conclusiones}

- La empresa, concebida como sistema abierto mantiene constante interacción con el entorno en el que se desenvuelve, depende del mismo e interactúa mediante la obtención de recursos para su operatividad y la entrega de productos y servicios que genera en su actividad.

- Como sistema, requiere de elementos que lo constituyen, los mismos que están relacionados y son interdependientes, su interacción funciona de tal manera que cualquier cambio, en cual- 
quiera de ellos, afecta a la totalidad, afecta al sistema.

- El sistema empresa tiene organización y estructura. La organización se encuentra definida por el conjunto de relaciones laborales, tecnológicas y económicas. Su estructura, se constituye en las interacciones de los elementos humanos, materiales, simbólicos (información) y energéticos (recursos financieros) que producen las relaciones, de tal manera que materializan la organización empresa.

- El origen de las causas de los problemas estructurales del sistema empresa está en la calidad de las relaciones laborales, relaciones tecnológicas y relaciones económicas que materializan la organización empresa mediante las interacciones de sus elementos constituyentes integrado por los recursos humanos, recursos materiales, la información y los recursos financieros, debido a que, concebida como sistema, cualquier cambio, en cualquiera de ellos, afecta al conjunto.

\section{Bibliografía}

Arévalo Bohórquez, L. E. (2013). La organización empresarial como sistema adaptativo complejo/Business organization as a complexity adaptative system/A organização empresarial como sistema adaptativo complexо. Estudios Gerenciales, 29(127), 258-265. Recuperado el 17 de 09 de 2015, de http://bibliotecavirtual.ups.edu.ec:2145/citedreferences/prod.academic_MSTAR_1497 175698/1BC350B42AE44C29PQ/ 7 ? accountid $=32861 \#$ top

Arias Galicia, F., \& Heredia Espinosa, V. (2012). Administración de Recursos Humanos. México: Litográfica Ingramex S.A.

Bertalanffy, L. (2007). Teoría General de los Sistemas. Buenos Aires: Fondo de Cultura Económica.

El Norte (19 de Mayo de 1997). De qué adolece el sistema administrativo? El Norte, 18. Recuperado el 21 de 09 de 2015, de http://bibliotecavirtual.ups.edu. ec:2145/docview/316189256/ abstract?accountid=32861\#top

González-Miranda, D. R. (2014). Los estudios organizacionales. Un campo de conocimiento comprensivo para el estudio de las organizaciones. Innovar 24(54), 43-58. Recuperado el 17 de 09 de 2015, de http:// bibliotecavirtual.ups.edu.ec:2145/ docview/1677001275/AF4072E73 1A64BCEPQ/2 ?accountid=32861

Hernández, S., \& Pulido , A. (2011). Fundamentos de Gestión Empresarial. México: McGraw Hill.

Laborda Castillo, L., \& De Zuani, E.R. (2005). Introducción a la Gestión Empresarial. Madrid: Servicio Publicaciones Universidad Alcalá.

Limone, A., \& Cademártori, D. (1998). La empresa una red de transformaciones. Santiago de Chile: Editorial Jurídica ConoSur Ltda.

Luhmann, N. (1997). Sociedad y sistema: 
la ambición de la teoría. Barcelona: Novagráfik, S. L.

Martínez, M. (2005). Ideas para el cambio y el aprendizaje para la organización . Bogotá: Ecoe Ediciones.

Maturana, H., \& Varela, F. (1996). El árbol del conocimiento. Madrid: Unigraf.

Mayntz, R. (1972). Sociología de la organización. Madrid: Gráfica Breogán.

Robbins, S. P., \& Coulter, M. (2010). Admi- nistración. México: Prentice-Hall.

Rodríguez Valencia, J. (2002). Estudio de Sistemas y procedimientos administrativos. México: International Thomson Editores.

Senge, P. (2006). La quinta disciplina en la práctica: estrategias y herramientas para construir la organización abierta al aprendizaje. Buenos Aires: Granica.

Recepción: 23-09-2015 - Aceptación: 03-12-2015. 